related to various physiological activities of cells such as ERK (14.9\%), PI3K (12.9\%), RTK-RAS (20.9\%) and other signaling pathways. There were 514 altered genes among all 205 patients: 491 mutations, 4 fusions, 10 copy number variants, and 9 deletions were found. The top 5 genes were TP53 (25.1\%), BRCA1 (7.4\%), ARIDIA (5.4\%), KRAS (4.7\%), and PIK3CA (3.5\%), etc. Ninety patients had homologous recombination repair (HRR) mutations and 71 patients had DDR mutations. RAD50 $(\mathrm{n}=4)$ or PALB2 ( $\mathrm{n}=3)$ mutations were associated with tumor mutational burden (TMB).

Conclusion: Mutations in genes of DDR pathway were closely associated in Chinese gynecological cancers patients, particularly the HRR pathway. The landscape of gene alteration and TMB among Chinese OC populations in this study will further assist the utilization of these biomarkers to individualized therapy strategies.

Oral (OMi7)

Gynecologic Cancer Screening

https://doi.org/10.3802/jgo.2021.32.S1.0Mi7

\section{The short-term impact of COVID-19 pandemic on cervical cancer screening: a systematic review and meta-analysis}

\section{Sabeena Sasidharan Pillai, ${ }^{1, *}$ Nagaraja Ravishankar ${ }^{2}$ \\ 'Manipal Institute of Virology, Manipal Academy of Higher Education, Manipal, India (sabeenauthradam@gmail.com) \\ ${ }^{2}$ Vallabhbhai Patel Chest Institute, New Delhi, India}

Objective: A systematic review and meta-analysis was performed to evaluate the short-term impact of severe acute respiratory syndrome coronavirus 2 on cervical cancer screening based on the number of eligible women screened before the pandemic and during the pandemic.

Methods: After ruling out registered or ongoing systematic review regarding the impact of coronavirus disease 2019 (COVID-19) infection in cervical cancer cases in PROSPERO database, the systematic review and meta-analysis protocol was registered in PROSPERO (CRD42021279305). The electronic databases were searched for articles published in English between January 2020 and September 2021 and the study was designed based on Preferred Reporting Items for Systematic Reviews and Meta-analyses guidelines updated in 2020. The meta-analysis component was modified appropriately to synthesise the pooled proportion of women attending cervical cancer screening before and during the pandemic. Studies reporting the number of women undergoing cervical cancer screening by cytology, human papillomavirus DNA testing or co-testing before the pandemic and during the pandemic were included. The studies reporting only the reduction in test volume or test rate per 100-person months were excluded. To assess the risk of bias in individual studies (quality assessment), chosen after the abstract and content review, the National Institutes of Health checklist for observational, cohort and cross-sectional studies was used. Meta-analysis was accomplished in STATA version 13.0 (College Station, TX, USA). The forest plots were constructed using metaprop package in STATA. A considerable amount of heterogeneity across the studies was anticipated as the included studies were mostly observational. The pooled proportion of women undergone cervical cancer screening was reported with $95 \%$ confidence interval (CI) along with $\chi^{2}$ statistic (Q statistic) and $\mathrm{I}^{2}$ index to quantify the heterogeneity.

Results: There are studies reporting the effect of COVID-19 on cervical cancer screening rate from Slovenia, Italy, Ontario (Canada), Scotland, Belgium, USA. The pooled proportion of women screened in 2019 was $8.79 \%$ (95\% CI=6.12\%-11.47\%). During the pandemic period, the pooled proportion of women undergone screening was $3.22 \%$ (95\% CI $=2.48 \%-3.96 \%)$. Conclusion: The decline in cancer screening rates has to be addressed to facilitate timely detection and management of precancerous lesions as well as cervical cancers. Scaling up of cervical cancer prevention strategies during the pandemic is essential to prevent the long-term effect of cervical cancer burden.

Oral (OMi8)

Miscellaneous

https://doi.org/10.3802/jgo.2021.32.S1.0Mi8

Which risk of malignancy index (RMI-1, 2, 3, 4) is better for differentiating adnexal masses: a diagnostic dilemma

\section{Vrushti Solanki, Pratibha Singh," Shashank Shekhar, Navdeep Kaur Ghuman, Garima Yadav, Priyanka Kathuria, Meenakshi Gothwal, Charu Sharma, Binit Sureka \\ All India Institute of Medical Sciences, New Delhi, India (drpratibha69@hotmail.com)}

Objective: To know the sensitivity and specificity of risk of malignancy index (RMI)-1, 2, 3, 4 to predict the nature of adnexal masses and to compare their areas under the curve (AUCs) and accuracy.

Methods: This is a prospective comparative study conducted in the Department of Obstetrics and Gynaecology at All India Institute of Medical Sciences, Jodhpur for 2 years from 2018 to 2020. This was the secondary objective of our study. All the women in the reproductive and post-menopausal age group presenting with adnexal masses, planned for surgery were 